\title{
Desnutrição: uma enfermidade presente no contexto hospitalar
}

\author{
Malnutrition: a disease found in the hospital setting
}

\author{
Tatiana Maria Palmeira dos Santos ${ }^{1} \bowtie$, Anne Karolyne dos Santos Silva ${ }^{2}$, Cynthia Barbosa Albuquerque dos Santos ${ }^{1}$, \\ Munik Samarah Gomes Souza ${ }^{1}$, Doriane da Conceição Lacerdas' ${ }^{1}$, José Alfredo dos Santos Júnior ${ }^{3}$, \\ Hugo José dos Santos Xavier ${ }^{1}$, Genildo Cavalcante Ferreira Júnior ${ }^{4}$ \\ ${ }^{1}$ Departamento de Nutrição da Universidade Tiradentes. Aracaju, SE, Brasil. \\ ${ }^{2}$ Hospital de Urgência de Sergipe. Aracaju, SE, Brasil. \\ 3 Departamento de Ciências Biológicas do Centro Universitário Cesmac. Maceió, AL, Brasil. \\ ${ }^{4}$ Pós-Graduação em Química e Biotecnologia, Universidade Federal de Alagoas. Maceió, AL, Brasil.
}

\section{RESUMO}

Objetivos: Analisar as associações entre o diagnóstico do estado nutricional pelos métodos objetivos e subjetivos, o período de hospitalização e a faixa etária de pacientes hospitalizados em um hospital público de Sergipe.

Métodos: Estudo transversal com adultos e idosos hospitalizados no período de julho a setembro de 2013. Foram investigados a faixa etária, o tempo de internação e métodos antropométricos de peso, circunferência da cintura, circunferência do braço, prega cutânea tricipital, índice de massa corporal, circunferência muscular do braço e área muscular do braço corrigida. A avaliação subjetiva do estado nutricional foi feita por meio da Avaliação Subjetiva Global e da Mini Avaliação Nutricional. Foram selecionados voluntários com alimentação exclusivamente por via oral. Para análise estatística aplicou-se o teste qui-quadrado de Pearson ou exato de Fisher e a correlação de Spearman, com nível de significância de $5 \%(\mathrm{p} \leq 0,05)$.

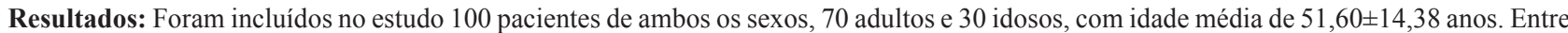
os pacientes avaliados, 57 (57\%) estavam internados há menos de 15 dias. Constatou-se prevalência de desnutrição de $58 \%$ pela circunferência do braço, $73 \%$ pela área muscular do braço corrigida e $69 \%$ pela circunferência muscular do braço. A avaliação subjetiva identificou desnutrição em 49 (49\%) dos indivíduos. Entre os idosos foram encontrados 13 (43,3\%) com sobrepeso de acordo com o índice da massa corporal.

Conclusões: Os parâmetros antropométricos e subjetivos de avaliação nutricional apresentaram divergências no diagnóstico final, confirmando a importância da utilização de mais de um método de avaliação para detecção de desnutrição hospitalar. Apesar da desnutrição ser prevalente em pacientes hospitalizados, o excesso de peso também foi encontrado, apontando para um processo de transição nutricional nesta população.

DESCRITORES: estado nutricional; pacientes internados; avaliação nutricional; transição nutricional.

\section{ABSTRACT}

Aims: To examine the associations between the diagnosis of nutritional status by objective and subjective methods, hospital length of stay, and the age of hospitalized patients in a public hospital in Sergipe, northeastern Brazil.

Methods: This is a cross-sectional study of adult and elderly patients hospitalized between July and September 2013. The following parameters were investigated: age, length of hospital stay, and anthropometric measurements, namely, waist circumference, midarm circumference, triceps skinfold, body mass index, arm muscle circumference, and corrected arm muscle area. The nutritional status was determined by subjective global assessment and mini-nutritional assessment. Volunteers who received exclusive oral feeding were selected. Pearson's chi-square test or Fisher's exact test and Spearman's correlation coefficient were used for the statistical analysis. The level of significance was set at 5\% ( $\mathrm{p} \leq 0.05)$. Results: A total of 100 patients, 70 adults and 30 elderly individuals, both male and female, with a mean age of $51.60 \pm 14.38$ years, were included in the study. Fifty-seven (57\%) patients were in hospital for less than 15 days. The prevalence of malnutrition was detected as follows: $58 \%$ by midarm circumference, $73 \%$ by corrected arm muscle area, and $69 \%$ by arm muscle circumference. The subjective assessment revealed that 49 (49\%) patients were suffering from malnutrition. Among elderly patients, 13 (43.3\%) were overweight, according to the body mass index. Conclusions: The anthropometric and subjective parameters used to assess nutritional status led to different diagnoses, thus confirming the importance to use more than one method for detecting malnutrition in the hospital setting. Although malnutrition is prevalent in hospitalized patients, overweight is also found, indicating nutrition transition in this population.

KEY WORDS: nutritional status; inpatients; nutritional assessment; nutritional transition. 
Abreviaturas: $\mathrm{AMBc}$, área muscular do braço corrigida; ASG, avaliação subjetiva global; $\mathrm{CC}$, circunferência da cintura; $\mathrm{CB}$, circunferência do braço; $\mathrm{CMB}$, circunferência muscular do braço; DCV, doenças cardiovasculares; IMC, índice de massa corporal; MAN, mini avaliação nutricional; OMS, organização mundial de saúde; PCT, prega cutânea tricipital; TI, tempo de internação.

\section{INTRODUÇÃO}

$\mathrm{O}$ estado nutricional do indivíduo reflete o equilíbrio entre a ingestão e as necessidades de nutrientes, as quais são influenciadas por diversos fatores. Os métodos utilizados para se avaliar o estado nutricional são objetivos e subjetivos, porém deve-se verificar qual o método mais sensível para permitir um diagnóstico nutricional mais preciso [1].

A desnutrição é problema de ampla consequência nos pacientes hospitalizados. Esse distúrbio está geralmente relacionado a um aumento da morbimortalidade e agravamento do estado clínico dos pacientes, pois acarreta deficiência de cicatrização de feridas, diminui a síntese de proteínas hepáticas e compromete o sistema imune, tornando-se fator de risco para infecções e contribuindo não só para prolongar o tempo de hospitalização, como também aumentar os custos hospitalares [2-4]. A prevalência de desnutrição em pacientes hospitalizados tem variado entre $2 \%$ a $8 \%$, dependendo da população estudada e do método utilizado $[5,6]$.

Estudos demonstram que após a internação hospitalar cerca de $70 \%$ dos pacientes que já eram inicialmente desnutridos sofrem agravamento do seu estado nutricional, o que contribui para o aumento da morbidade e mortalidade em até $65 \%$ dos pacientes. A desnutrição em pacientes hospitalizados está relacionada a doenças e fatores associados (infecções, doenças do trato gastrointestinal, renais e hepáticas, complicações cirúrgicas e debilidade musculoesquelética), ou decorre de ingestão alimentar inadequada durante o período de internação. Neste contexto, destaca-se a importância da detecção precoce do risco nutricional, através da triagem nutricional, por meio de um método efetivo de triagem em até 72 horas da admissão do paciente, predizendo, assim, a desnutrição e desfechos negativos e possibilitando cuidados nutricionais adequados $[7,8]$.

Por outro lado, estudos recentes têm constatado um aumento da prevalência de sobrepeso e obesidade na população, fato este justificado pela transição nutricional caracterizada por modificações no padrão de nutrição e de consumo, como consequência das transformações econômicas, sociais e demográficas [9-12].

Diante do exposto, este estudo teve como objetivo analisar as associações entre o diagnóstico do estado nutricional pelos métodos objetivos e subjetivos, o período de hospitalização e a faixa etária de pacientes hospitalizados em um hospital público do estado de Sergipe.

\section{MÉTODOS}

Este estudo transversal incluiu adultos e idosos, deambulantes ou não, de ambos os sexos, internados no setor de clínica médica de um hospital público de urgências de Aracaju, Sergipe, que atende pacientes de todas faixas etárias, gêneros e patologias. Os dados foram obtidos durante o período de julho a setembro de 2013. Todos os pacientes incluídos assinaram um termo de consentimento livre e esclarecido. O estudo seguiu os preceitos éticos estabelecidos na Resolução 466/12 do Conselho Nacional de Saúde, sendo aprovado pelo Comitê de Ética em Pesquisa em Seres Humanos da Universidade Tiradentes (protocolo no 020513R).

Para a inclusão dos participantes, foram considerados pacientes adultos e idosos com condições físicas e mentais que permitissem as avaliações necessárias. Não foram levados em consideração o tipo de doença ou o motivo da hospitalização. Não foram incluídos na amostra pacientes com deformidade física, edema periférico, ascite ou anasarca, com amputação ou paralisia de membros, pacientes em terapia nutricional por via parenteral ou aqueles que se recusaram a assinar o termo de consentimento. A amostra foi não probabilística, por conveniência, e buscou a participação de todos os internados no período do estudo que preenchessem os critérios de inclusão.

A antropometria foi realizada através das medidas de peso, estatura, circunferência da cintura (CC), circunferência do braço $(\mathrm{CB})$ e prega cutânea tricipital (PCT). O peso e a estatura foram utilizados para o cálculo do índice de massa corporal (IMC) e a CB e a PCT para cálculo da circunferência muscular do braço (CMB) e área muscular do braço corrigida ( $\mathrm{AMBc}$ ). As medidas de IMC, CC, CB e PCT são indicadores de gordura corporal, enquanto que a $\mathrm{CMB}$ e a $\mathrm{AMBc}$ refletem a reserva corporal de massa magra.

Nos pacientes que deambulavam, o peso corporal foi medido com os indivíduos descalços e em trajes 
leves, mediante utilização de uma balança eletrônica portátil (Filizola, São Paulo, SP), com precisão de $100 \mathrm{~g}$ e capacidade de $150 \mathrm{~kg}$. A estatura nos adultos foi obtida por um estadiômetro portátil (Avanutri, Três Rios, RJ), com precisão de $1 \mathrm{~mm}$ e capacidade de $2 \mathrm{~m}$, com os indivíduos descalços, em posição ortostática [13]. Para os idosos e os pacientes que não deambulavam, foram estimados peso e estatura de acordo com Chumlea et al. [14,15]. A altura do joelho (AJ), necessária para o cálculo da estimativa do peso e da estatura, foi aferida com o indivíduo em posição supina, com a perna direita formando um ângulo de noventa graus com o joelho e o tornozelo, utilizandose paquímetro (Cescorf, Porto Alegre, RS), constituído por uma parte fixa, que foi posicionada na superfície plantar do pé (calcanhar) e uma parte móvel, que foi pressionada sobre a cabeça da patela (rótula).

O IMC foi calculado a partir da razão entre o peso $(\mathrm{kg})$ e a estatura (m) ao quadrado. Para classificação do estado nutricional pelo IMC foram utilizados os pontos de corte propostos pela Organização Mundial da Saúde (OMS) [16] para os adultos e Lipschitz [17] para os idosos. A CC, com o objetivo de avaliar a adiposidade abdominal em função da sua associação com risco de doenças cardiovasculares (DCV), foi medida com fita métrica inelástica no ponto médio entre o último arco costal e a crista ilíaca e classificada de acordo com a OMS $[13,18]$. A CB foi medida com fita métrica inelástica no ponto médio entre o acrômio e o olécrano, com o braço relaxado; a PCT com adipômetro científico do tipo Lange ${ }^{\circledR}(0$ a $60 \mathrm{~mm} \times 1 \mathrm{~mm})$, na parte posterior do braço, na altura do ponto médio medido para a CB. Ambas as medidas foram classificadas por percentis de acordo com Frisancho [19] para adultos e National Health and Nutrition Examination Survey (NHANES) III [18] para os idosos [9,20].

A medida de CMB, formada pela associação de outras medidas (CB e PCT), levando em consideração o formato cilíndrico do braço, foi calculada a partir da equação proposta e classificada por percentis de acordo com Frisancho [19] para adultos e de acordo com NHANES III [18] para os idosos. A medida de $\mathrm{AMBc}$ foi determinada a partir do resultado da $\mathrm{CMB}$ e calculada conforme a fórmula proposta por Heymsfield et al. [21] e classificada por percentis de acordo com Frisancho [19].

A avaliação do estado nutricional foi realizada também através de métodos subjetivos: Avaliação Subjetiva Global (ASG) proposta por Detsky [22] para os pacientes adultos e a Mini Avaliação Nutricional (MAN), de acordo com Guigoz et al. [23] para os pacientes idosos.
As variáveis quantitativas foram descritas por média, mediana e desvio padrão e as variáveis categóricas por frequências absolutas e relativas. Para avaliar a associação entre as variáveis, aplicou-se o teste qui-quadrado de Pearson ou exato de Fisher. $O$ nível de significância adotado foi de $5 \%(\mathrm{p} \leq 0,05)$.

\section{RESULTADOS}

Foram avaliados 100 pacientes, sendo 70 adultos e 30 idosos, 69 homens e 31 mulheres. A idade dos pesquisados variou de 24 a 81 anos, com média de $51,6 \pm 14,3$ anos e mediana de 50 anos. De acordo com o tempo de internação (TI) (dicotomizado em $>15$ dias e $\leq 15$ dias), a maioria, 57 (57\%) permaneceram internados por menos de 15 dias. Os diagnósticos clínicos encontrados foram insuficiência renal crônica 38 (38\%), neoplasia 25 (25\%), trauma cranioencefálico $13(13 \%)$, acidente vascular cerebral $13(13 \%)$ e traumas $11(11 \%)$.

Através da avaliação antropométrica do grupo total pelo IMC, CC e PCT, a maioria estava eutrófica. Entretanto, de acordo com $\mathrm{CB}$, $\mathrm{AMBc}$ e $\mathrm{CMB}$, predominou a desnutrição. Houve associação do estado nutricional avaliado por todos os parâmetros antropométricos com a faixa etária no grupo total $(p<0,05)$. Segundo avaliação dos dados por faixa etária, verificou-se de acordo com o IMC que 23 $(68,6 \%)$ dos adultos estavam eutróficos, 65 (92,9\%) foram classificados sem risco cardiovascular e mais da metade apresentou baixa reserva muscular de acordo com $\mathrm{CB}, \mathrm{CMB}$ e AMBc. Entre os idosos estudados foram encontrados $13(43,3 \%)$ com sobrepeso de acordo com o IMC. O acúmulo de gordura abdominal, que caracteriza risco para doença cardiovascular, foi encontrada nos 16 idosos em que foi possível a realização da medida; e a medida da CMB foi mais sensível para detectar baixa reserva muscular (Tabela 1).

No que diz respeito às avaliações subjetivas (ASG e MAN), metade dos indivíduos adultos estava moderadamente desnutrida e um número elevado de idosos, 14 (46,7\%) encontravam-se desnutridos. Todas as categorias do estado nutricional nos métodos subjetivos de acordo com a faixa e no grupo total apresentaram associação significativa $(\mathrm{p}<0,05)$ (Tabela 1).

Relacionando-se o estado nutricional com o TI por faixa etária, houve associação significativa apenas com o IMC. Entre os idosos, quanto maior o TI, maior foi o percentual de desnutridos. Dentre os pacientes 
idosos desnutridos pelas avaliações da $\mathrm{CB}, \mathrm{CMB}$, $\mathrm{AMBc}$ e MAN, a maior parte tinha TI acima de 15 dias (Tabela 2).

Relacionando-se o estado nutricional da avaliação objetiva com os métodos subjetivos de tria- gem nutricional por faixa etária, houve associação significativa da maioria dos parâmetros antropométricos (IMC, CC, $\mathrm{CMB}$ e $\mathrm{AMBc}$ ) com os métodos subjetivos da ASG, nos adultos, e da MAN, nos idosos (Tabela 3).
Tabela 1. Classificação dos dados antropométricos de pacientes hospitalizados segundo a faixa etária. Aracaju, Sergipe, 2013.

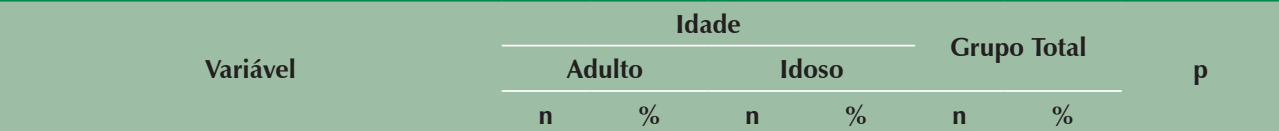

Classificação do IMC

$\begin{array}{lccccccc}\text { Eutrófico } & 48 & 68,6 & 07 & 23,3 & 55 & 55 & <0,001^{*}+ \\ \text { Magreza } & - & - & 10 & 33,3 & 10 & 10 & \\ \text { Desnutrição } & 9 & 12,8 & - & - & 9 & 9 & \\ \text { Sobrepeso } & 13 & 18,6 & 13 & 43,3 & 26 & 26 & \\ \text { Total } & 70 & 100,0 & 30 & 100,0 & 100 & 100,0 & \\ \text { Classificação da CC } & & & & & & & \\ \text { Adequada } & 65 & 92,9 & - & - & 65 & 75,6 & <0,001 * \neq \\ \text { Risco de DCV } & 5 & 7,1 & 16 & 100 & 21 & 24,4 & \\ \text { Total } & 70 & 100,0 & 16 & 100,0 & 86 & 100,0 & \\ \text { Classificação da CB } & & & & & & & \\ \text { Eutrófico } & 19 & 27,2 & 19 & 63,3 & 38 & 38 & <0,001 *+ \\ \text { Desnutrição } & 50 & 71,4 & 8 & 26,7 & 58 & 58 & \\ \text { Risco de sobrepeso } & - & - & 2 & 6,7 & 2 & 2 & \\ \text { Sobrepeso } & 1 & 1,4 & 1 & 3,3 & 2 & 2 & \\ \text { Total } & 70 & 100,0 & 30 & 100,0 & 100 & 100,0 & \end{array}$

Classificação da PCT

\begin{tabular}{lccccccc} 
Eutrófico & 43 & 61,4 & 20 & 66,7 & 63 & 63 & $0,001^{*+}$ \\
Risco nutricional & - & - & 04 & 13,3 & 14 & 14 & \\
Desnutrição & 10 & 14,3 & 04 & 13,3 & 4 & 04 & \\
Obesidade & 17 & 24,3 & 01 & 3,3 & 18 & 18 & \\
Sobrepeso & - & - & 01 & 3,3 & 1 & 01 & \\
Total & 70 & 100,0 & 30 & 100,0 & 100 & 100,0 & \\
Classificação da AMBc & & & & & & & \\
Desnutrição leve/moderada/grave & 62 & 88,6 & 11 & 36,7 & 73 & 73 & $<0,001^{*+}$ \\
Eutrófico & 8 & 11,4 & 19 & 63,3 & 27 & 27 & \\
Total & 70 & 100,0 & 30 & 100,0 & 100 & 100,0 & \\
Classificação da CMB & & & & & & & \\
Eutrófico & 16 & 22,9 & 15 & 50 & 31 & 31 & $<0,001^{*+}$ \\
Baixa massa magra & 54 & 77,1 & 15 & 50 & 69 & 69 & \\
Total & 70 & 100,0 & 30 & 100,0 & 100 & 100,0 & \\
Classificação da ASG ou MAN & & & & & & & \\
Nutrido & 35 & 50 & 16 & 53,3 & 51 & 51 & $<0,001^{*+}$ \\
Moderadamente desnutrido & 35 & 50 & - & - & 35 & 35 & \\
Desnutrido & - & - & 14 & 46,7 & 14 & 14 & \\
Total & 70 & 100,0 & 30 & 100,0 & 100 & 100,0 & \\
\hline
\end{tabular}

* Associação significativa.

+ Teste Exato de Fisher.

\# Qui-quadrado de Pearson.

IMC: índice de massa corporal; CC: circunferência da cintura; CB: circunferência do braço; PCT: prega cutânea tricipital AMBc: área muscular do braço corrigida; CMB: circunferência muscular do braço; ASG, avaliação subjetiva global MAN: miniavaliação nutricional. 
Tabela 2. Classificação dos dados antropométricos de pacientes adultos e idosos hospitalizados segundo o tempo de internação. Aracaju, Sergipe, 2013.

\begin{tabular}{|c|c|c|c|c|c|c|c|c|c|c|c|c|c|}
\hline \multirow{4}{*}{ Variável } & \multicolumn{12}{|c|}{ Tempo de internação (dias) } & \multirow{4}{*}{$\mathbf{p}^{*}$} \\
\hline & \multicolumn{4}{|c|}{ Adulto } & \multicolumn{4}{|c|}{ Idoso } & \multicolumn{4}{|c|}{ Grupo Total } & \\
\hline & \multicolumn{2}{|c|}{$\leq 15$ dias } & \multicolumn{2}{|c|}{$>15$ dias } & \multicolumn{2}{|c|}{$\leq 15$ dias } & \multicolumn{2}{|c|}{$>15$ dias } & \multicolumn{2}{|c|}{$\leq 15$ dias } & \multicolumn{2}{|c|}{$>15$ dias } & \\
\hline & n & $\%$ & n & $\%$ & n & $\%$ & n & $\%$ & n & $\%$ & $\mathbf{n}$ & $\%$ & \\
\hline \multicolumn{14}{|l|}{ Classificação do IMC } \\
\hline Eutrófico & 26 & 61,9 & 22 & 78,6 & 04 & 26,7 & 03 & 20,0 & 30 & 52,6 & 25 & 58,1 & $0,027+$ \\
\hline Magreza & - & - & - & - & 01 & 6,7 & 09 & 60,0 & 01 & 1,8 & 09 & 20,9 & \\
\hline Desnutrição & 06 & 14,3 & 03 & 10,7 & - & & - & - & 06 & 10,5 & 03 & 7,0 & \\
\hline Sobrepeso & 10 & 23,8 & 03 & 10,7 & 10 & 66,7 & 03 & 20,0 & 20 & 35,1 & 06 & 14,0 & \\
\hline Total & 42 & 100,0 & 28 & 100,0 & 15 & 100,0 & 15 & 100,0 & 57 & 100,0 & 43 & 100,0 & \\
\hline \multicolumn{14}{|l|}{ Classificação da CC } \\
\hline Adequada & 37 & 88,1 & 28 & 100 & - & - & - & - & 37 & 68,5 & 28 & 87,5 & 0,085 \\
\hline Risco de DCV & 05 & 11,9 & - & - & 12 & 100 & 04 & 100 & 17 & 31,5 & 04 & 12,5 & \\
\hline Total & 42 & 100,0 & 28 & 100,0 & 12 & 100,0 & 04 & 100,0 & 54 & 100 & 32 & 100 & \\
\hline \multicolumn{14}{|l|}{ Classificação da CB } \\
\hline Eutrófico & 13 & 31,0 & 06 & 21,4 & 10 & 66,7 & 09 & 60,0 & 23 & 40,3 & 15 & 34,8 & 0,222 \\
\hline Desnutrição & 29 & 69,0 & 21 & 75,0 & 02 & 13,3 & 06 & 40,0 & 31 & 54,4 & 27 & 62,8 & \\
\hline Risco de sobrepeso & - & - & - & - & 02 & 13,3 & - & - & 02 & 3,5 & - & - & \\
\hline Sobrepeso & - & - & 01 & 3,6 & 01 & 6,7 & - & - & 01 & 1,8 & 01 & 2,4 & \\
\hline Total & 42 & 100,0 & 28 & 100,0 & 15 & 100,0 & 15 & 100,0 & 57 & 100,0 & 43 & 100,0 & \\
\hline \multicolumn{14}{|l|}{ Classificação da PCT } \\
\hline Eutrófico & 23 & 54,8 & 20 & 71,4 & 11 & 73,3 & 09 & 60,0 & 34 & 59,6 & 29 & 67,4 & 0,200 \\
\hline Risco nutricional & - & - & - & - & - & - & 04 & 26,7 & - & - & 04 & 9,3 & \\
\hline Desnutrição & 06 & 14,3 & 04 & 14,3 & 02 & 13,3 & 02 & 13,3 & 08 & 14,0 & 06 & 14,0 & \\
\hline Obesidade & 13 & 31,0 & 04 & 14,3 & 01 & 6,7 & - & - & 14 & 24,6 & 04 & 9,3 & \\
\hline Sobrepeso & - & & - & - & 01 & 6,7 & - & - & 01 & 1,8 & - & - & \\
\hline Total & 42 & 100,0 & 28 & 100,0 & 15 & 100,0 & 15 & 100,0 & 57 & 100,0 & 43 & 100,0 & \\
\hline \multicolumn{14}{|l|}{ Classificação da CMB } \\
\hline Eutrófico & 09 & 27,3 & 07 & 25,0 & 10 & 66,7 & 05 & 33,3 & 19 & 33,3 & 12 & 27,9 & 0,399 \\
\hline Baixa massa magra & 33 & 78,6 & 21 & 75,0 & 05 & 33,3 & 10 & 66,7 & 38 & 66,7 & 31 & 72,1 & \\
\hline Total & 42 & 100,0 & 28 & 100,0 & 15 & 100,0 & 15 & 100,0 & 57 & 100 & 43 & 100 & \\
\hline \multicolumn{14}{|l|}{ Classificação da AMBc } \\
\hline Eutrófico & 05 & 11,9 & 03 & 10,7 & 11 & 73,3 & 08 & 53,3 & 16 & 28,1 & 11 & 25,6 & 0,547 \\
\hline Desnutrição leve/moderada/grave & 37 & 88,1 & 25 & 89,3 & 04 & 26,7 & 07 & 46,7 & 41 & 71,9 & 32 & 74,3 & \\
\hline Total & 42 & 100,0 & 28 & 100,0 & 15 & 100,0 & 15 & 100,0 & 57 & 100,0 & 43 & 100,0 & \\
\hline \multicolumn{14}{|l|}{ Classificação da ASG ou MAN } \\
\hline Nutrido & 19 & 45,2 & 16 & 57,1 & 10 & 66,7 & 06 & 40,0 & 29 & 50,9 & 22 & 51,2 & 0,416 \\
\hline Moderadamente desnutrido & 23 & 54,8 & 12 & 42,9 & - & - & - & - & 23 & 40,3 & 12 & 27,9 & \\
\hline Desnutrido & - & - & - & - & 05 & 33,3 & 09 & 60,0 & 05 & 8,8 & 09 & 20,9 & \\
\hline Total & 42 & 100,0 & 28 & 100,0 & 15 & 100,0 & 15 & 100,0 & 57 & 100,0 & 43 & 100,0 & \\
\hline
\end{tabular}

* Qui-quadrado de Pearson.

+ Associação significativa.

IMC: índice de massa corporal; CC: circunferência da cintura; CB: circunferência do braço; PCT: prega cutânea tricipital; AMBc: área muscular do braço corrigida; CMB: circunferência muscular do braço; ASG, avaliação subjetiva global; MAN: miniavaliação nutricional. 
Tabela 3. Avaliação da classificação dos dados antropométricos de pacientes adultos e idosos hospitalizados segundo avaliação subjetiva global e mini-avaliação nutricional. Aracaju, Sergipe, 2013.

\begin{tabular}{|c|c|c|c|c|c|c|c|c|c|c|c|}
\hline \multirow{3}{*}{ Variável } & \multicolumn{4}{|c|}{ ASG } & \multicolumn{4}{|c|}{ MAN } & \multirow{2}{*}{\multicolumn{2}{|c|}{ Grupo Total }} & \multirow{3}{*}{ Valor de $\mathbf{p}^{*}$} \\
\hline & \multicolumn{2}{|c|}{ Nutrido } & \multicolumn{2}{|c|}{$\begin{array}{l}\text { Desnutrição } \\
\text { Moderada/grave }\end{array}$} & \multicolumn{2}{|c|}{ Nutrido } & \multicolumn{2}{|c|}{ Desnutrido } & & & \\
\hline & n & $\%$ & n & $\%$ & n & $\%$ & $\mathbf{n}$ & $\%$ & n & $\%$ & \\
\hline \multicolumn{12}{|l|}{ Classificação do IMC } \\
\hline Eutrófico & 25 & 71,4 & 23 & 65,7 & 6 & 37,5 & 1 & 7,1 & 55 & 55,0 & $<0,001+$ \\
\hline Magreza & - & & - & & 4 & 25,0 & 6 & 42,9 & 10 & 10,0 & \\
\hline Desnutrição & 5 & 14,3 & 4 & 11,4 & - & & - & & 9 & 9,0 & \\
\hline Sobrepeso & 5 & 14,3 & 8 & 22,9 & 6 & 37,5 & 7 & 50,0 & 26 & 26,0 & \\
\hline Total & 35 & 100,0 & 35 & 100,0 & 16 & 100,0 & 14 & 100,0 & 100 & 100,0 & \\
\hline \multicolumn{12}{|l|}{ Classificação da CC } \\
\hline Adequada & 34 & 97,1 & 31 & 88,6 & - & & - & & 65 & 75,6 & $<0,001+$ \\
\hline Risco de DCV & 1 & 2,9 & 4 & 11,4 & 9 & 100 & 7 & 100 & 21 & 24,4 & \\
\hline Total & 35 & 100,0 & 35 & 100,0 & 09 & 100,0 & 07 & 100,0 & 86 & 100,0 & \\
\hline \multicolumn{12}{|l|}{ Classificação da CB } \\
\hline Eutrófico & 9 & 25,7 & 10 & 28,6 & 10 & 62,5 & 9 & 64,2 & 38 & 38,0 & 0,113 \\
\hline Desnutrição & 25 & 71,4 & 25 & 71,4 & 4 & 25,0 & 4 & 28,6 & 58 & 58,0 & \\
\hline Risco de sobrepeso & - & - & - & - & 1 & 6,3 & 1 & 7,1 & 2 & 2,0 & \\
\hline Sobrepeso & 1 & 2,9 & - & - & 1 & 6,3 & - & & 2 & 2,0 & \\
\hline Total & 35 & 100,0 & 35 & 100,0 & 16 & 100,0 & 14 & 100,0 & 100 & 100,0 & \\
\hline \multicolumn{12}{|l|}{ Classificação da PCT } \\
\hline Eutrófico & 20 & 77,1 & 23 & 54,7 & 07 & 58,3 & 13 & 72,3 & 63 & 63,0 & 0,082 \\
\hline Risco nutricional & - & - & - & - & - & - & 04 & 22,2 & 04 & 4,0 & \\
\hline Desnutrição & - & - & 10 & 23,8 & 4 & 33,4 & - & - & 14 & 14,0 & \\
\hline Sobrepeso & - & - & - & - & - & - & 1 & 5,5 & 1 & 1,0 & \\
\hline Obesidade & 8 & 22,9 & 9 & 21,5 & 1 & 8,3 & - & - & 18 & 18,0 & \\
\hline Total & 28 & 100,0 & 42 & 100,0 & 12 & 100,0 & 18 & 100,0 & 100 & 100,0 & \\
\hline \multicolumn{12}{|l|}{ Classificação da CMB } \\
\hline Eutrófico & 11 & 31,4 & 5 & 14,3 & 6 & 37,5 & 9 & 64,3 & 31 & 31,0 & $<0,001+$ \\
\hline Baixa massa magra & 24 & 68,6 & 30 & 85,7 & 10 & 62,5 & 5 & 35,7 & 69 & 69,0 & \\
\hline Total & 35 & 100,0 & 35 & 100,0 & 16 & 100,0 & 14 & 100,0 & 100 & 100,0 & \\
\hline \multicolumn{12}{|l|}{ Classificação da AMBC } \\
\hline Desnutrição leve/moderada/grave & 28 & 80,0 & 34 & 97,1 & 5 & 31,2 & 6 & 42,9 & 73 & 73,0 & $<0,001+$ \\
\hline Eutrófico & 7 & 20,0 & 1 & 2,9 & 11 & 68,8 & 8 & 57,1 & 27 & 27,0 & \\
\hline Total & 35 & 100,0 & 35 & 100,0 & 16 & 100,0 & 14 & 100,0 & 100 & 100,0 & \\
\hline \multicolumn{12}{|l|}{ Idade } \\
\hline Adulto & 35 & 100 & 35 & 100 & - & - & - & - & 70 & 70,0 & $<0,001+$ \\
\hline Idoso & - & & - & & 16 & 100 & 14 & 100 & 30 & 30,0 & \\
\hline Total & 35 & 100,0 & 35 & 100,0 & 16 & 100,0 & 14 & 100,0 & 100 & 100,0 & \\
\hline
\end{tabular}

* Qui-quadrado de Pearson.

+ Associação significativa.

ASG, avaliação subjetiva global; MAN: mini avaliação nutricional; IMC: índice de massa corporal; CC: circunferência da cintura; CB: circunferência do braço; PCT: prega cutânea tricipital; CMB: circunferência muscular do braço; AMBc: área muscular do braço corrigida. 


\section{DISCUSSÃO}

Analisando-se o grupo total no presente estudo, a maioria era eutrófica de acordo com o IMC, CC e PCT e desnutrida pela CB, AMBc e CMB. Houve associação da antropometria com a faixa etária. Observou-se a menor frequência de desnutrição quando considerado o IMC como indicador, fato semelhante ao estudo de Arruda et al. [24], com maioria do sexo masculino, onde o IMC identificou o menor número de indivíduos desnutridos [24]. Ressalta-se que a condição de desnutrição pode se desenvolver durante a hospitalização $[25,26]$. Contudo, quando analisados os pacientes idosos, isoladamente, observou-se um elevado percentual de desnutridos segundo o diagnóstico pelo IMC, resultado semelhante ao estudo de Schuindt e Andrade com [27]. É importante mencionar que alguns estudos mencionam as limitações do IMC para a avaliação nutricional na prática clínica e que, em pacientes hospitalizados não é indicado o IMC como um método isolado de avaliação; o ideal é a associação de vários métodos $[1,28]$.

É imprescindível destacar o elevado percentual de pacientes com excesso de peso na amostra estudada, especialmente entre os idosos, fato semelhante aos estudos de Schuindt e Andrade [27] e de Crestani et al. [10]. Em concordância com estes achados, Bosco et al. [1], ao avaliar o IMC em 100 adultos e idosos hospitalizados, revelaram semelhança de frequências de eutrofia e excesso de peso $(47,0 \%$ vs $48,0 \%)$ [1]. Esses autores relatam que a prevalência de excesso de peso encontrada segue uma tendência mundial juntamente com outros estudos que têm demonstrado mudança no perfil nutricional da população brasileira, com aumento da prevalência de obesidade, caracterizando-se como um grave problema de saúde pública $[1,27,29]$. Entretanto, afirmam que apesar dos estudos revelarem um aumento do excesso de peso tanto em adultos quanto em idosos hospitalizados, a desnutrição proteico-calórica ainda é uma realidade nos hospitais públicos [1].

Analisando-se a população de idosos, observouse no presente estudo que a maioria estava com risco de DCV, segundo a medida da CC. Estes dados corroboram com dados recentes sobre a transição nutricional. Silva et al. [30] acrescentam ainda que o excesso de peso acarreta vários tipos de doenças, e esses pacientes terão maior risco metabólico, maior possibilidade de complicações e maior permanência hospitalar [30]. O excesso de peso é encontrado com grande frequência em todos os grupos de renda e em todas as regiões brasileiras [31].
Foi importante verificar que, entre os índices antropométricos de adiposidade (IMC, CC, CB e PCT) analisados, a CB foi a única que detectou desnutrição na maioria dos adultos, mostrando-se, portanto, mais sensível para detecção precoce da desnutrição nessa faixa etária. Observou-se também que a CMB mostrou-se bom indicador da depleção muscular e correlacionou-se com os índices de adiposidade em ambas as faixas etárias. A depleção da massa magra é muito importante, pois em situações como inanição e estresse prolongado, as reservas proteicas são mobilizadas para atender à demanda da fase aguda e das proteínas secretoras [32]. Já a PCT detectou um significativo número de pacientes com excesso de gordura, resultados condizentes com outros estudos que vêm demonstrando aumento da prevalência de obesidade, como já comentado acima [33].

Relacionando-se o estado nutricional com o TI por faixa etária, neste estudo houve associação significativa apenas com o IMC. Nos adultos eutróficos, a maioria tinha menor TI e, dentre os idosos com magreza, a maior parte tinha maior TI. A partir destes achados, verifica-se que o IMC, apesar das suas limitações já citadas anteriormente, neste estudo mostrou-se bom preditor do TI, tanto nos adultos quanto nos idosos.

Foi importante verificar também que, dentre os pacientes idosos desnutridos, pelas avaliações da $\mathrm{CB}$, $\mathrm{CMB}, \mathrm{AMBc}$ e MAN, a maior parte tinha também maior TI, mostrando-se estes, assim, bons parâmetros para predizer TI nos idosos. Outros estudos já constataram que a $\mathrm{CB}$ era um bom marcador de TI e recomendaram o uso desta como método de escolha para avaliar o estado nutricional e inferir TI, pois a $\mathrm{CB}$ é um método fácil de mensurar e menos oneroso que outros equipamentos $[24,30]$.

É consenso que quanto maior o TI, maior possibilidade de o paciente ficar desnutrido e, consequentemente, aumentar ainda mais a permanência hospitalar. Esse círculo vicioso acaba acarretando mais problemas para os pacientes e mais gastos para o serviço de saúde [6,34]. Pacientes em risco nutricional têm um tempo de hospitalização, em média, 50\% maior que o de pacientes saudáveis; além disso, a hospitalização por mais de 15 dias está associada a um risco três vezes maior de desnutrição $[35,36]$.

De acordo com a ASG, nos adultos, foi encontrado um elevado número de pacientes moderadamente desnutridos, resultado semelhante ao estudo de Crestani et al. [10]. Já nos idosos, a MAN revelou percentual elevado de desnutridos semelhante à pesquisa de Monteiro et al. [37]. Relacionando métodos objetivos 
e subjetivos de avaliação do estado nutricional, Araújo et al. [29] e Schuindt e Andrade [27] demonstraram também boa correlação da ASG com a antropometria em pacientes internados. Já no estudo realizado por Yamauti et al. [38], que incluiu ASG e os indicadores antropométricos CB, PCT e IMC, a ASG revelou maior número de desnutridos quando comparada à avaliação objetiva, dado semelhante ao deste estudo. Por esta razão, a ASG possibilita a identificação de pacientes com risco de desnutrição antes que ocorra mudança nas medidas antropométricas [38].
Conclui-se que os parâmetros antropométricos e subjetivos de avaliação nutricional apresentaram divergências no diagnóstico final, confirmando a importância da utilização de mais de um método de avaliação para detecção de desnutrição hospitalar. Percebe-se também que, apesar da desnutrição ser prevalente em pacientes hospitalizados, o excesso de peso também é encontrado nesta população. Assim, é essencial a implementação de atividades de orientação nutricional que enfoquem também a obesidade e não somente a subnutrição.

\section{REFERÊNCIAS}

1. Bosco VEF, Sousa KMM, Viana KDAL, Calado IL. Estado nutricional de indivíduos hospitalizados em um hospital universitário de São Luís, Maranhão. Rev Pesq Saúd. 2013;14(1):21-26.

2. Sampaio RMM, Vasconcelos CMCS, Pinto FJM. Prevalência de desnutrição segundo a avaliação nutricional subjetiva global em pacientes internados em um hospital público de Fortaleza (CE). Rev Baiana Saúde Públ. 2010;34(2):311-20.

3. Fidelix MSP, Santana AFF, Gomes JR. Prevalência de desnutrição hospitalar em idosos. Rev Assoc Bras Nutr. 2013;5(1):60-8.

4. Fonseca DA, Garcia RRM, Stracieri APM. Nutritional profile of patients with Neoplasms according to diferent indicators. Rev Dig de Nutr. 2009;3(5):444-61.

5. Dobner T, Telles CT, Pomatti G, Pasqualotti A, Bettinelli LA. Avaliação do estado nutricional em pacientes renais crônicos em hemodiálise. Sci Med. 2014;24(1):11-8. http://dx.doi.org/10.15448/1980-6108.2014.1.15858

6. Maciel JRV, Oliveira CJR, Tada CMP. Associação entre risco de disfagia e risco nutricional em idosos internados em hospital universitário de Brasília. Rev Nutr Campinas. 2008;21(4):411-21. http://dx.doi.org/10.1590/s1415-52732008000400005

7. Carvalho IL, Ramos ICM. Triagem Nutricional em paciente adulto. Fundação Hospitalar do Estado de Minas Gerais. Diretrizes Clínicas. Protocolos Clínicos. 2014; p.1-7.

8. Aquino RC, Philippi ST. Identificação de fatores de risco de desnutrição em pacientes internados. Rev Assoc Med Bras. 2011;57(6):637-43. http://dx.doi.org/10.1590/S0104-42302011000600009

9. Reis VB, Urbano LS, Freiberg CK, Hernandes SCM. Perfil nutricional de pacientes hospitalizados em um hospital geral do município de São Paulo. Nutrição Brasil. 2010;9(1):5-11.

10. Crestani N, Bieger P, Milani RELK, Dias RDAL, Alscher S, Lienert RSC. Perfil nutricional de Paciente adultos e idosos admitidos em um Hospital Universitário. Ciênc Saúde, 2011;4(2):45-9.

11. Almeida JC, Kuhmmer R, Sponchiado E, Laflor CM, Weber B. Desenvolvimento de um instrumento de triagem nutricional para avaliação do risco de desnutrição na admissão hospitalar. Rev Bras Nutr Clin. 2008;23(1):28-33.

12. Cintra RMGC, Garla P, Bosio M C, Tognoli M, Soares AS, Matto MS et al. Estado nutricional de pacientes hospitalizados e sua associação com o grau de estresse das enfermidades. Rev Simbio-Logias. 2008;1(1):145-56.

13. Rossi L, Caruso L, Galante AP. Avaliação Nutricional: Novas Perspectivas. São Paulo: Roca / Centro Universitário São Camilo; 2008.

14. Chumlea WC, Guo SS, Steinbaugh ML et al. Prediction of stature from knee height for black and white adults and children with application to mobility-impaired or handicapped persons. J Am Diet Assoc. 1994;94(12):1385-8. http://dx.doi.org/10.1016/0002-8223(94)92540-2

15. Chumlea WC, Guo S, Wolihan K, Cockran D, Kuczmarsk RJ, Johnson CL et al. Stature prediction equations for elderly non-hispanic white, non-hispanic black, and mexican-american person developed from NHANES III data. J Am Diet Assoc. 1998;98(2):137-42. http://x.doi. org/10.1016/S0002-8223(98)00036-4

16. World Health Organization (WHO). WHO Technical Report Series. Pshysical Status: the use and interpretation of anthropometry. Geneva: WHO, 1995. 452p.

17. Lipschitz DA. Screening for nutricional status in the elderly. Primary care. 1994;21(1):55-67.

18. World Health Organization (WHO). Obesity- Preventing and managing the global epidemic. Report of a WHO consultation on obesity. Geneva, 1998.

19. Frisancho AR. Anthropometric Standards for the Assessment of Growth and Nutritional Status. Ann Arbor: University of Michigan Press, 1990.

20. Kuczmarski MF, Kuczarisk RJ, Najjar M. Descriptive anthropometric reference data for older Americans. J Am Diet Assoc. 2000;100:59-66. http://dx.doi.org/10.1016/S0002-8223(00)00021-3

21. Heymsfield SB, McManus C, Smith J, Stevens V, Nixon DW. Anthropometric measurement of muscle mass: revised equations for calculating bone-free arm muscle area. Am J Clin Nutr. 1982;36:680-90.

22. Detsky AS, McLaughlin JR, Baker JP, Johnston N, Whittaker S, Mendelson RA, et al. What is subjective global assessment of nutritional status? JPEN. 1987;11:8-13. http://dx.doi.org/10.1177/014860718701100108 
23. Guigoz Y, Vellas B, Garry PJ. Mini Nutritional Assessment: a practical assessment tool for grading the nutritional state of elderly patients. Facts Res Gerontol. 1994;(suppl 2):15-59.

24. Arruda CV, Pinho CPS, Santos ACO. Repercussões nutricionais em pacientes portadores de insuficiência cardíaca associada à miocardiopatia no Nordeste Brasileiro. Nutr Clin Diet Hosp. 2014;34(3):37-47. http://dx.doi.org/10.12873/343vitadearruda

25. Raslan M, Gonzalez MC, Gonçalves MCD, Colagrossi FPB, Cecconello I, Waitzberg DL. Applicability of nutritional screening methods in hospitalized patients. Rev Nutr. 2008;21(5):553-61.

26. Rodrigues CC, Leandro-Merhi VA, Oliveira MRM, Fogaça K P. Predominância de excesso de peso entre pacientes hospitalizados independente do tempo de internação. Rev Bras Nutr Clin. 2008;23(4):256-61.

27. Schuindt PS, Andrade AHG. Perfil nutricional de pacientes internados em um hospital de Londrina-Paraná. Anais do IV Forum Científico da Faculdade de Apucarana [Internet]. Apucarana, Paraná; 2012. [cited 2015 jan]. Available from: http://www.fap.com.br/forum 2012/forum/pdf/ Saude/Comunicacao_Oral/ResSauCO10.pdf

28. Cortez ACL, Martins MCC. Indicadores Antropométricos do Estado Nutricional em Idosos: Uma Revisão Sistemática. UNOPAR Cient Ciên Biol Saúde. 2012;14(4):271-7.

29. Araújo MAR, Lima LS, Ornelas GC, Logrado MHG. Análise comparativa de diferentes métodos de triagem nutricional do paciente internado. Comun Ciênc Saúde. 2010;21(4):331-42.

30. Silva HGV, Santos SO, Silva NO, Ribeiro FD, Josua LL, Moreira ASB. Circunferência do braço: bom preditor de tempo de internação hospitalar. Ceres: Nutr Saúde. 2011;6(2);95-104.

31. IBGE. Pesquisa de orçamentos familiares 2008-2009. Despesas, rendimentos e condições de vida. Rio de Janeiro: IBGE; 2010.224 p.

32. Vannucchi H, Unamuno MRL, Marchini JS. Avaliação do estado nutricional. Medicina, Ribeirão Preto. 1996;29:5-18. http://dx.doi.org/10.11606/ issn.2176-7262.v29i1p5-18

33. BRASIL, Ministério da Saúde. Vigitel, Brasil 2013: Vigilância de fatores de risco e proteção para doenças crônicas não transmissíveis por meio de inquérito telefônico. Brasília: Ministério da Saúde; 2014. 120 p.

34. Miranda RNA, Salgado NA, Almeida TTG, Silva TCS, Maciel AP. Métodos de avaliação para a detecção de Desnutrição em idosos Hospitalizados atendidos pelo Programa de Residência Multiprofissional em Saúde do Idoso, Belém/PA. XXII Congresso Brasileiro de Nutrição e III Congresso Ibero-Americano de Nutrição, 2012.

35. Ferreira IT. Desnutrição no internamento hospitalar: implicações econômicas [dissertation]. [Porto]: Faculdade de Ciências da Nutrição da Universidade do Porto; 2009.

36. Mello ED, Texeira LB, Beghetto MG, Luft VC. Desnutrição Hospitalar cinco anos após a IBRANUTRI. SBNPE-Sociedade Brasileira de Nutrição Parenteral e Enteral, Hospital das Clínicas de Porto Alegre. Rev Bras Nutr Clin. 2003;18(2):65-9.

37. Monteiro AMP, Dias FV, Ferreira AVM, Santos LC, Oliveira DR. Déficit nutricional em pacientes geriátricos atendidos em um hospital de pronto-socorro, em Belo Horizonte-MG. Rev Mineira Enferm. 2010;3(4):541-9.

38. Yamauti AK, Ochiai PS, Moab AA, Rosiris R A, Ribeiro RHC, Barretto ACP. Avaliação Nutricional Subjetiva Global em Pacientes Cardiopatas. Arq Bras Cardiol. 2006;87(6):772-7. http://dx.doi.org/10.1590/S0066-782X2006001900014 C 